\title{
Reply to Letter to Editor
}

\section{Femoral Component Positioning in Hip Resurfacing with and Without Navigation}

\author{
Pascal-André Vendittoli MD, MSc, $\operatorname{FRCS}(\mathrm{C})$, \\ Muthu Ganapathi MSc, FRCS(Orth), \\ Martin Lavigne MD, MSc, FRCS(C)
}

Published online: 24 March 2009

(C) The Association of Bone and Joint Surgeons 2009

We acknowledge Dr. Andrea Emilio Salvi for his general comments and opinions on hip resurfacing surgical technique and factors that may affect clinical outcome. Even if no specific question or comment is directly related to our study [2], we will try to provide a scientific input on the points raised.

The first "procedural trap" reported by Dr. Salvi is in relation to the femoral component stem (providing initial stability and alignment). In the resurfacing system used in our study (Durom ${ }^{\circledR}$; Zimmer, Inc, Warsaw, IN), the femoral stem is smooth and slightly smaller than the drilled hole. The purpose of the stem is purely to allow centering the femoral component over the prepared femur to allow uniform cement mantle $(0.75-1.0 \mathrm{~mm}$ in this system). The stem is intentionally undersized to avoid load transfer to the bone and prevent femoral head osteopenia by stress shielding. We agree femoral head cylindrical reaming done over a single guide wire may lead to error in the preparation. However, in the resurfacing system we have used, the femoral head preparation is done over a stiff rod inserted in a drilled femoral channel, which reduces/avoids such error. So any error is likely to have happened in the initial guidewire placement and not during the final head preparation.

We totally agree an accurate preoperative plan is mandatory to select optimal femoral position and orientation and implant size. However, as shown in our study, even

P.-A. Vendittoli ( $₫)$, M. Lavigne

Department of Surgery, Maisonneuve-Rosemont Hospital,

University of Montreal, 5415 Boul L'Assomption, Montreal

H1T 2M4, QC, Canada

e-mail: pa.vendittoli@ videotron.ca

M. Ganapathi

Department of Orthopaedics, Maisonneuve-Rosemont Hospital, University of Montreal, Montreal, QC, Canada with preoperative planning, without computer navigation, we did not reach a precision of $\pm 5^{\circ}$ in $38 \%(n=33)$ of the cases versus $0 \%$ with surgical navigation. As mentioned in our article, it is not known if increasing our precision will have an effect on early and long-term implant survivorship.

Regarding the second trap, Dr. Salvi proposes a slightly angulated cemented component may present some motion and finally create a fracture by repeated edge loading. In our experience and understanding of the current literature, component loosening usually does not preclude femoral neck fracture. Femoral neck fracture occurs in the early postoperative period and may be related to weakening of the femoral neck by a surgically created notch (mainly in the superior zone), femoral component implanted in varus $\left(<130^{\circ}\right)$, presence of a cyst in the femoral head-neck junction, femoral component left proud (leaving cancellous bone exposed at the superior part of the femoral head), and femoral neck osteopenia. In these fracture cases, bone in the femoral component will still be solidly fixed to the implant. When progressive change in the femoral component orientation occurs later, this should be viewed as a femoral head loosening. In these cases, intracomponent femoral bone will show lysis and often necrosis. Regarding femoral neck notching, to create a significant defect, in a case where femoral head-neck offset is preserved, significant error in angulation should have happened. One way to prevent such an error is to perform a sequential femoral head reaming, starting $4 \mathrm{~mm}$ larger than the planned size. Room for additional bone removal should be assessed before downsizing to the next reamer.

The third trap refers to postoperative femoral neck narrowing. There is no clear understanding in the current literature regarding the etiology of femoral neck narrowing reported by some authors. In our experience with the 
Durom ${ }^{\circledR}$ system, we did not find any cases of femoral neck narrowing $(>10 \%)$ in more than 200 cases at a minimum of 3 years followup (range, 3-6 years). Femoral neck narrowing may be attributable to simple bone remodeling associated with newer orientation of femoral loading (mainly increased valgus compared with the native neckshaft angle), load transfer through the femoral stem when press fit or cemented, reaction secondary to exaggerated metal ion production, neck impingement on the metallic acetabular component, and so on. One thing is clear: femoral neck narrowing is not universal for all surgeons and for all resurfacing systems. Additional research should be done to better understand the mechanism and determine whether occurrence should be considered a complication or simply a normal process after hip resurfacing.

Without clear scientific answers from the literature, we do not believe an unfixed central femoral stem should be pointed to as a significant cause for bone resorption in current cemented designs. However, we agree the necessity of its presence can be questioned once the initial alignment is aided particularly in a cementless femoral design. Recently, Amstutz and Le Duff reported improved shortterm survivorship by cementing the femoral head metaphyseal stem [1]. It is yet to be determined whether the presence of a fixed stem (cemented or cementless) would improve middle and long-term survival of hip resurfacing components or create femoral stress shielding and late component loosening.

\section{References}

1. Amstutz HC, Le Duff MJ. Cementing the metaphyseal stem in metal-on-metal resurfacing: when and why. Clin Orthop Relat Res. 2009;467:79-83.

2. Ganapathi M, Vendittoli PA, Lavigne M, Günther KP. Femoral component positioning in hip resurfacing with and without navigation. Clin Orthop Relat Res. 2008 May 17. [Epub ahead of print]. 